\section{Introduction to the Encyclopedia of Law and Data Science: 'Directions for Use'}

\author{
The concept, its authors and the ways \\ of reading $i^{1}$
}

Normally a book does not require instructions for its reading. Readers enjoy their liberty to read it from the beginning, to jump here and there across chapters and paragraphs or to be guided by its table of contents to perform a cherry-picking reading. It is the beauty of books and of the freedom of their users. Nevertheless, I feel some words and 'directions for use' are appropriate in this case.

The Encyclopedia of Law and Data Science, comprising 64 entries of various forms and length, along with many other 'hidden' ones inside other entries (e.g. definitions and terms defined within entries and captured in the analytical index), is the fruit of the efforts of $\mathbf{6 7}$ contributors from three continents whose expertise converged from many varied fields: from law and data science especially, but also from economics, statistics, computer engineering, physics, biomedical engineering and history, philosophy, neuro-engineering, political science and geo-informatics. Despite this wealth of experiences and amplitude of topics contemplated, we cannot claim completeness, and this can only be considered a first attempt in reaching the ambitious mapping and bridging goals this multi-authored endeavour envisages.

Indeed, the Encyclopedia of Law and Data Science aims to bring together jurists, computer scientists and data analysts to uncover the challenges, opportunities and fault lines that arise as these groups are increasingly thrown together by expanding attempts to regulate and adapt to a data-driven world. It is an ambitious project aiming at mapping concepts and tools at the crossroads of the many disciplines involved in data science and for law in particular. An intellectual exercise with practical implications for

1 This encyclopedia is partially produced in the framework and with the contribution of the 'Legality Attentive Data Scientists' H2020 project Grant Agreement No. 956562. Sincere thanks go to Sara Canduzzi, Danielle Da Costa Leite Borges, Camilla Signoretta for their precious help in managing the editorial revision of this work. a multitude of users: from domain experts to policymakers, from businesses to practitioners.

Contributors coming together from many countries have developed the ability to interact among themselves striving to share their languages and integrate the respective background domain knowledge in one shared idiolect that they want now to share with a wider audience: many contributors come from joint international academic experiences and experts working at regulatory authorities thus also intersecting with a variety of stakeholder experiences.

The list of contributors enclosed in the Encyclopedia attests the worldwide contributions gathered here. It is obvious that many other entries could have been added and might need to be covered in the future. Nevertheless, we decided to move forward with this first edition of the Encyclopedia since the need for adjustments and innovations will emerge continuously in this sparkling interdisciplinary field of knowledge. Indeed, science in the subject domains is evolving fast as is the related regulation. As a regulatory example, only when the manuscript was ready for the publisher did the EC release its Proposal for a Regulation on a European approach for Artificial Intelligence. ${ }^{2}$

As jurists begin to spearhead attempts to regulate data-driven industry, they often deploy concepts and terms - such as 'discrimination', 'bias' or 'consent' - whose meaning may be agreed upon in the legal field but is difficult to translate in the data analysis context. At the same time, as data scientists are progressively tasked with the actualization - the modelling and implementation - of legal rules, they may employ concepts - like 'data protection', 'integrity' or 'portability' - whose meaning is similarly settled within the analytics field, but difficult to transpose into juridical terms or have very specific meanings in the legal sphere. Therefore, the Encyclopedia aims at creating a comparative and cross-disciplinary place of mutual understanding, providing definitions of shared concepts that set the theoretical framework and the practical implementation template of a common language for co-processing and joint-controlling key notions for both data scientists and jurists, thereby answering the specific need to clarify and operationalize

\footnotetext{
${ }^{2}$ https://digital-strategy.ec.europa.eu/en/library/ proposal-regulation-european-approach-artificialintelligence.
} 
these newly introduced notions in the digital economy.

Because of this state of affairs, it is necessary to explore the ways in which meanings diverge and converge between these disciplines to set a truly common field of knowledge, language and expertise.

With that goal in mind, the Encyclopedia of Law and Data Science attempts to create a comparative and interdisciplinary lexicon that draws experts from many fields to define important cross-over concepts from their many technical angles. This is one key reason for which many entries unfold as stand-alone texts with their own narrative, originality and normative insights. Many contain definitions and conceptual explanations which, in themselves, constitute a part of an evolving lexicon navigable via the analytical index. Indeed, the engineering of these varied contributions produced a multilayered output which adds to the 'traditional' ways to enjoy the reading (going by entry or reading the entire book) a different port of entry by searching the analytical index.

Having these definitions and conceptual explanations emerge in titled paragraphs in the individual entries would have broken the internal narrative of the entries and would have hindered the interested reader's navigation of the entries. On the other hand, the analytical index was conceived as a guide or a map to the explanatory treasures embedded in the entries. Metaphorically, it offers a mirror image of the table of contents as an alternative, alphabetical, larger list of entries for surfing through and across the entire lexicon.

The creation of the shared lexicon as such, we think, gives a striking tone of originality to the book as whole, as it aims to initiate a true common cross-disciplinary place of mutual understanding, building an idiolect for data science, thoroughly bridging hard and social sciences. An idiolect is a linguistic pattern regarded as unique among speakers of a person's language or dialect. It is not just vocabulary; it includes everything in between from how we put words together to what we imagine they mean. Therefore, the Encyclopedia helps domain experts understand the use others make of the same phraseology in their own domain.

Although a number of initiatives have gathered experts from very different domains, including informatics, law and broadly speaking data science, none has attempted to build a common language, nor has it done as this
Encyclopedia aims to do. Here, legal experts do not only put the accent of law on data science but also integrate their knowledge with those of data scientists and vice versa.

Such an endeavour would have been an unrealistic ambition without the leverage of many settled international and interdisciplinary collaborations especially, but not exclusively, between jurists, data scientists and computer engineers.

\section{Embroiling the common narrative}

Data scientists are the key players of the data economy for their ability to both unlock the insights of data and tell a remarkable story about and from them. Yet, these skills need to be bridged with the ability to fully understand the legal, ethical, economic, and technological implications and constraints of extracting knowledge from data.

Information are data, knowledge is the ability to effectively put them into use. Thus, knowledge extraction is not only mining of new data, but is the generation of useful data (see the entries Data Mining and Clustering; Argument Mining). Knowledge extraction acquires value by the way its results are used.

The process is well known; data mining does not move from a hypothesis but seeks to generate hypotheses, to find correlations, the socalled patterns. Discovered patterns turn into statements describing relationships among certain data; only when we assume those relationships as decisive factors in our activities, do we transform them into knowledge because we use them. To a large extent what is relevant is not the discovered patterns that simplify the enumeration of all the facts in a data set but considering them as knowledge to be used even when their results are not or cannot be explained. ${ }^{3}$ Already this fact raises ethical, technical and legal issues. ${ }^{4}$ For instance, patterns are readily

\footnotetext{
${ }^{3}$ See the entry Explainability.

4 See Giovanni Comandé, 'The Rotting Meat Error: From Galileo to Aristotle in Data Mining?' (2018) 4(3) European Data Protection Law Review 270 277; T. Hagendorff, 'The Ethics of AI Ethics: An Evaluation of Guidelines' (2019), arXiv, 1-15. On the ethical implications of AI-only declarations and principles the literature is vast, but the following is a start: AI Now Institute; Association for Computing Machinery (ACM) with its Committee on Professional Ethics (https://ethics.acm.org/
} 
transformed into models and individuals are pigeonholed in them, often under the shadow of personalizing a service, while shaping their decision-making context by selecting the information they see, as advertisements or search results on a search engine on the internet for instance. ${ }^{5}$ Awareness of the potential uses of data science outputs is a must for data scientists that will increasingly shape their activities.

Of course, data scientists are very aware that patterns are not true across all the data and their truthfulness depends on several factors (e.g. integrity of the data, size of the sample,

2018-code-draft-2/); Public Policy Council (https://acm.org/public-policy/usacm); the socalled Asilomar Principles of the Future of Life Institute (https://futureoflife.org/ai-principles/); Foundation for Responsible Robotics (http:// responsiblerobotics.org); Google's AI Principles (https://www.blog.google/technology/ai/aiprinciples); Institute of Electrical and Electronics Engineers (IEEE) Global Initiative on Ethics of Autonomous and Intelligent Systems (IEEE Global Initiative on Ethics of Autonomous and Intelligent Systems, 'Ethically Aligned Design: A Vision for Prioritizing Human Well-being with Autonomous and Intelligent Systems, Version 2', http:// standards.ieee.org/develop/indconn/ec/autono mous_systems.html); OpenAI (https://openai. com/); Partnership on AI (a partnership on AI industry-led set up by Google, Apple, Facebook, Amazon, IBM, Microsoft, https://www.partner shiponai.org/); Software and Information Industry Association (SIIS) (SIIS, 'Ethical Principles for Artificial Intelligence and Data Analytics', 2017, http://www.siia.net/LinkClick.aspx?fileticket= b46tNqJuiJA $\% 3 \mathrm{~d} \&$ tabid $=577 \&$ portalid $=0 \&$ mid=17113); World Economic Forum's Center for the Fourth Industrial Revolution (https://www. weforum.org/center-for-the-fourth-industrialrevolution/areas-of-focus). See also https:// ethicsinaction.ieee.org/. In the specific domain of the administration of justice, see European Commission for the Administration of Justice (CEPEJ) (2018), 'European Ethical Charter on the Use of Artificial Intelligence in Judicial Systems and Their Environment', https://rm.coe.int/ ethical-charter-en-for-publication-4-december2018/16808f699c.

5 See in general Fundamental Rights Agency, 'Getting the Future Right: Artificial Intelligence and Fundamental Rights', 2020, https://fra. europa.eu/sites/default/files/fra_uploads/fra-2020artificial-intelligence_en.pdf8. See the examples in AlgorithmWatch, 'Automating Society Report 2020', https://automatingsociety.algorithmwatch. org/wp-content/uploads/2020/12/AutomatingSociety-Report-2020.pdf. sources, statistical relevance). They are aware that these different degrees of truthfulness and relevance may contain or lead to 'bias', 'discrimination' and 'disparate impacts' on individuals. ${ }^{6}$ Yet, very often, progress in data science is oblivious to how the inferences it generates need to be balanced with an understanding of the risks that this very progress creates for society and individuals, democracy ${ }^{7}$ and fundamental rights. ${ }^{8}$ In the same vein data scientists' skills need to be linked with the ability to develop applications based on data in such a way that regulations do not unreasonably prevent the deployment of technologies while technologies comply with ethical and legal requirements. ${ }^{9}$

All these issues have emerged in many projects with data scientists with different expertise and nationality and are firmly present in the literature. Indeed, this collective work stems from a global constellation of connections and collaborations across a number of research projects, training programmes and intellectual interplays. The entire lexicon is partially the output of the H2020 funded project 'Legality Attentive Data Scientists' ${ }^{10}$ and constitutes its initial mapping of conceptual gaps among disciplines involved in the data-driven society and produces a clear glossary to reduce misunderstandings and impracticability of adopted technical/legal solutions.

Most of data science developments are based on the analysis by machine learning and other artificial intelligence (AI) methods of huge amounts of data, including users' preferences. Researchers must be aware that the analyses of users' practices, modelling their preferences or

${ }^{6}$ See the corresponding entries along with Algorithmic Discrimination; Algorithmic Fairness; Scoring.

7 European Group on Ethics in Science and New Technologies (2018), 'Statement on Artificial Intelligence, Robotics and "Autonomous Systems",, https://ec.europa.eu/research/ege/pdf/ ege_ai_statement_2018.pdf. Electronic copy available at: https://ssrn.com/abstract $=3414805$ proposed a set of basic principles and democratic prerequisites, based on the fundamental values laid down in the EU Treaties and in the EU Charter of Fundamental Rights.

${ }^{8}$ See, for instance, the entries Bias; Ethics. On a specific domain, see CEPEJ (n 4).

${ }^{9}$ Concerns emerge in many entries, but see Accountability; Confidentiality; Access; Anonymity.

10 LeADS: Grant Agreement No. 956562. www. legalityattentivedatascientists.eu. 
their behaviour, require access to personal data, which carries ethical considerations as well as the need for new ways to deal with security threats. ${ }^{11}$ Technical advancements and the skyrocketing computational ability question the borderlines between personal and non-personal data. ${ }^{12}$ For these reasons many entries directly or indirectly touch on personal data protection. ${ }^{13}$ Several entries are expressly dedicated to it (Data Protection; Data Protection Impact Assessment; Data Subject; Forgotten (Right to Be); Lawfulness and Necessity; Public Interest; Legitimate Interest; Portability; Transparency).

More generally, data are at the centre of any AI development and deployment fuelled by machine learning and big data. Following the High-Level Expert Group on Artificial Intelligence, ${ }^{14}$ any trustworthy AI unfolding from them must be (1) lawful, complying with all applicable laws and regulations (2) ethical, ensuring adherence to ethical principles and values and (3) robust, to prevent unintentional harm (see the entry Liability).

These three components are strictly interrelated and require the appropriate bridges we start to build with this collective endeavour. ${ }^{15}$ In addition, even the Guidelines for Trustworthy AI do not explicitly deal with trustworthy AI's first component (lawfulness), a gap that needs to be covered beginning with a shared understanding of notions we initiate here. For this reason, a number of entries, without losing in-depth and technical precision,

11 See the entry Cybersecurity.

12 See, for instance, the entries Anonymity; Pseudonymization; Mobility. See also Gianclaudio Malgieri and Giovanni Comandé, 'Sensitiveby-Distance: Quasi-Health Data in the Algorithmic Era' (2017) 26(3) Information and Communications Technology Law 229.

13 See, for example, the entries Data Breach; Supervisory Authorities.

14 High-Level Expert Group on Artificial Intelligence, 'Ethics Guidelines for Trustworthy AI' (European Commission 2019), https://ec.europa.eu/newsroom/dae/document.cfm?doc_id=58477. For a rather critical comment, see $\mathrm{M}$. Veale, 'A Critical Take on the Policy Recommendations of the EU High-Level Expert Group on Artificial Intelligence' (2020) European Journal of Risk Regulation, doi:10/ ggjdjs.

15 See also Giovanni Comandé, 'Unfolding the Legal Component of Trustworthy AI: A Must to Avoid Ethics Washing' (2020) Annuario di Diritto Comparato e di Studi Legislativi 39-62. illustrate such fundamental notions to a multidisciplinary audience (Anonymity; Computer Programs; Cybersecurity; Data Breach; Data Quality; Machine Learning; Privacy-Preserving Technologies; Privacy by Design) stressing their transdisciplinary meaning while making it accessible and relevant across disciplines.

This effort to open the richness of data science also to experts in other domains is sometimes performed in joint writings (for example, Healthcare (Data Science in); Mobility Data) and in other instances is matched with the determination to grant access to notions from a more legal perspective (e.g. Informed Consent; Freedom of Information; Freedom of Expression; Access to Public Data; Governance (of Personal Data Flows); Open Data and Public Sector Information; Patents; Trade Secrets; Proportionality) or with a more policy oriented attitude (e.g. Surveillance; Fairness; Unfairness). Many other entries cannot be classified precisely because of their nature and the way they are elaborated express a truly transversal attitude (e.g. Privacy; Predictive Analytics; Profilel Profiling; Reasonable Safeguards; Erasure; Decision-making; Artificial General Intelligence).

Without expressly addressing the question in a specific entry ${ }^{16}$ the entire work faces the key question: why should AI require a different (harsher?) treatment than other technologies which 'benefited' from years of loose legal frameworks for decades? ${ }^{17}$

The many entries address some of the features of AI induced by big data and data science, explaining how AI enables the exploitation of several unseen vulnerabilities that have not (yet) triggered a legal reaction (beyond race, gender, sex habits, nationality, etc.), and it can do so in a massive, personalized way.

Similarly, the entries set the framework to understand how AI offers the opportunity to exploit specific biases, individual and even transient vulnerabilities, to establish contingent and lasting asymmetries of powers. Emphasis is on the shared characteristic of every AI solution that makes it different to a

16 However, see the entries Artificial General Intelligence; Algorithmic Discrimination; Algorithmic Fairness.

17 See for instance D. Bose, M. Segui-Gomez and J. R. Crandall, 'Vulnerability of Female Drivers Involved in Motor Vehicle Crashes: An Analysis of US Population at Risk' (2011) CI, 12 American Journal of Public Health 2368-2373. 
similar solution which is solely human based: the former is faster in acting and can act upon a larger number of information and variables than those available to the humans interacting with them. Understanding these characteristics does not lead to our stressing the threats versus the opportunities they offer. On the contrary, by raising awareness of the risks of downplaying the role of regulation this work favours a faster and sounder uptake of the technologies involved.

Prevention of harm is an ethical imperative for AI that requires the utmost attention to prevent malicious uses (see the entry Ethics); it also requires us to search for guiding criteria to push legal rules to be up to speed with technological developments and business models. As ever larger parts of modern life become digitized, individuals generate an increasing volume and variety of digital traces, which reveal information about their everyday activity and movements exposing especially those that are most vulnerable (Children (in the Digital Environment); Consent; Vulnerability).

In the fast-evolving scenario of the datadriven society, security must evolve on an ongoing basis and the security management process should be flexible enough to adapt quickly to the organization of security to address new threats. Similarly legal rules need to be flexible and open enough to enable technological innovation mindful of fundamental rights.

Facing such challenges, we hope this collective work helps in developing a data science capable of maintaining its innovative solutions within the borders of law - by design and by default - while at the same time helping expand the legal frontiers in line with innovation needs, preventing the enactment of legal rules technologically unattainable. However, the crossfertilization of scientific cultures remains one of the Encyclopedia's flagship features: generating a much-needed - and currently absent lexicon to define important crossover concepts; enabling the cross-fertilization of scientific cultures generating a much-needed - and currently absent - multilevel, multipurpose common understanding of concepts useful for (future) researchers, policymakers, software developers, lawyers, and market players.

\section{Talking to the readers}

As anticipated, there is a clear gap in the language used by the scientific and professional communities called to work together in our expanding data-driven societies. Jurists and data scientists (statisticians, computer engineers, computer scientists, etc.) face two conceptual and linguistic challenges. On the one hand, they are called to work hand in hand to bring to existence, deploy and regulate the unfolding of the big data and AI revolution in many fields, but they lack a common language, an idiolect that makes the dialogue and the daily working together possible. On the other hand, they share several words used in their activities, but these words echo different concepts in the different domains leading to confusion and unsettling. The Encyclopedia aims at filling these gaps, coalescing together in one community the many experts needed to innovate, regulate and adapt to a data-driven world.

Benefits of the Encyclopedia are many.

On one side, data scientists currently tend to have little knowledge of the legal implications and limitations of their activities, let alone of the language they are using. This is especially true in the light of the cross-border nature of information technology, which requires an approach that can deal with differences in legislation. For instance, from a legal point of view, some of the difficulties in drafting policies about data, such as standards and regulations, but also terms and conditions of enterprises, depend on the lack of knowledge that policymakers have about the technical tools and methodologies to operate on data. For this reason, policymaking generally requires a long and iterative interaction between data scientists and legal experts, which in turn can be problematic due to knowledge asymmetries and the difficulties that the two categories face in communicating.

From a different angle, establishing communication channels requires us to address at the same time academics and practitioners working with topics related to law and technology and comparative law studies, policymakers and legislators along with data science academics, computer scientists and software engineers. In its practical approach, the Encyclopedia also aims at reaching out to professionals and to civil servants, who are often required to draw inspiration from foreign experiences to develop their own policies regarding the regulation of new technologies. All will benefit from having such a tool at hand.

We are aware that addressing such a diverse audience with a single instrument might sound like wishful thinking; indeed, bringing together 
such a large, global and varied group of prominent experts appeared to be a mission impossible. And yet here it is: the Encyclopedia of Law and Data Science. We are deeply convinced it can and will help to pursue not only lawfulness of data mining and AI development, but both the fullest protection for fundamental rights and, simultaneously, the largest possible data exploitation in the digital economy we are living in.

Giovanni Comandé 